\title{
Hypokalaemia: Improving the investigation, management and therapeutic monitoring of hypokalaemic medical inpatients at a district general hospital
}

\author{
Mark Jordan, Jenny Caesar \\ Wrexham Maelor Hospital, UK
}

\begin{abstract}
Hypokalaemia is prevalent in $20 \%$ of hospitalised patients. Furthermore, inadequate management of hypokalemia was identified in $24 \%$ of these patients. Associated with significant patient morbidity and mortality, the identification, investigation, and treatment of hypokalaemia was identified as an area for improvement in the management of medical inpatients.
\end{abstract}

The project aims to measure the assessment, management, and therapeutic monitoring of medical inpatients with hypokalaemia in a district general hospital.

All medical inpatients over a one week period who met the criteria for hypokalaemia (serum potassium $<3.5 \mathrm{mmol} / \mathrm{L}$ on standard biochemical sample) were included in the audit. Patient's notes were located and evaluated to identify if they had mild, moderate, or severe hypokalaemia. Further data on ECG requests, repeat U\&Es, serum magnesium analysis, treatment prescribed, and medication review dates was collated. A re-audit was completed after the introduction of a set of interventions which included a hypokalaemia treatment algorithm.

Pre-intervention analysis of all medical inpatients, who met our inclusion criteria for hypokalaemia, identified 32 patients. 25 of these patients met the criteria for mild hypokalaemia (3.1-3.4 mmol/L) and 7 met the criteria for moderate hypokalaemia $(2.5-3.0 \mathrm{mmol} / \mathrm{L})$. Only $7 / 32(22 \%)$ patients were receiving adequate treatment based on trust guidelines.

Post intervention results showed marked improvement in the management of patients with hypokalaemia. A total of 30 patients were identified in this post-intervention group. There were $16 / 30$ patients who qualified as mild hypokalaemia (3.1-3.4 mmol/L) and 14/30 with moderate hypokalaemia (2.5-3.0 mmol/L). 19/30 (63\%) patients in the post-intervention group were correctly prescribed appropriate medication doses consistent with the treatment algorithm.

Following the initial success of the project, analysis at 3 months showed a positive trend for sustained improvement when compared to the preintervention results.

\section{Problem}

High incidence of hypokalaemia was noted amongst acute medical inpatients during ward rounds at a 700 bed district general hospital. Although potassium replacement medication use was widespread, inconsistency in appropriate dosing, drug preparations, and the monitoring of treatment responses was evident.

With no local guidelines on the management of hypokalaemia, informal survey of the junior doctors highlighted uncertainty of appropriate investigation and management within our hospital. Consequently, haphazard potassium replacement regimes were in place leading to patient safety concerns, especially for inadequate management of dangerously low potassium values. Medication reviews to address potential causes for hypokalaemia were not routine and ECGs infrequently requested to assess for arrhythmic complications in moderate to severe hypokalaemia. Additionally, lack of serum blood monitoring and clear medication review dates increased the risk of patients remaining on potassium replacement beyond acceptable time frames, predisposing to hyperkalaemia.

Service evaluation and quality improvement was required to improve patient safety and standardise the treatment of hypokalaemia.

\section{Background}

The normal range of plasma potassium is $3.5-5.1 \mathrm{mmol} / \mathrm{l}$. Daily potassium requirements of a $70 \mathrm{~kg}$ male are $70 \mathrm{mmol}$ compared to a $40 \mathrm{~kg}$ woman who will need $40 \mathrm{mmol}$. Hypokalaemia is the most common electrolyte abnormality in hospitalised patients. When defined as a value of less than $3.5 \mathrm{mmol} / \mathrm{L}$, hypokalaemia is found in $20 \%$ of hospital inpatients, but occurs in only $2 \%$ of otherwise healthy adults (1-3). Approximately $5 \%$ of hospitalised patients with confirmed hypokalaemia have serum potassium concentrations of $<3.0 \mathrm{mmol} / \mathrm{l}$ (4). Additionally, up to $50 \%$ of patients with normal potassium on admission may develop hypokalaemia during their inpatient stay (5). 


\section{BMJ Quality Improvement Reports}

Deviations to both extremes (hypo- and hyperkalaemia) are related to an increased risk of cardiac complications. Potassium levels below $3.0 \mathrm{mmol} / \mathrm{l}$ may cause significant Q-T interval prolongation with subsequent risk of torsade des pointes, ventricular fibrillation, and sudden cardiac death (6). Those at particular risk are patients with cardiac ischemia, heart failure, and left ventricular hypertrophy, in whom even mild-to-moderate hypokalaemia increases the likelihood of cardiac arrhythmias (2). For example, the mortality rate of hospitalised hypokalaemic patients with cardiovascular disease was 10 -fold higher than the general hospitalised population (7).

Hypokalaemia can be life-threatening when severe $(<2.5 \mathrm{mmol} / \mathrm{L})$ but is usually well tolerated in otherwise healthy people and patients are often asymptomatic. Consequently, the diagnosis is easily missed and recognition of high risk patients, frequent laboratory monitoring, and prompt treatment is essential to reduce the incidence of hypokalaemic complications (8). Risk of hypokalemia in patients taking diuretics is higher in the elderly and enhanced by concomitant illness, such as heart failure or nephrotic syndrome (9). Latrogenic causes are also important to consider. Of patients taking non-potassium-sparing diuretics, $10 \%$ to $40 \%$ develop hypokalemia (9). Other morbidities associated with a higher incidence of hypokalemia include; $21 \%$ of hospitalised patients with AIDS, $12.6 \%$ of hospitalised patients with alcoholism, and patients after bariatric surgery (10-12).

Inadequate management of hypokalaemia is identified in $24 \%$ of hospitalised patients (13). Common complications associated with severe hypokalaemia include thirst $(43 \%)$, paraesthesias $(38 \%)$, muscle weakness (14\%), and muscle cramps (7\%) (4). The incidence of ventricular fibrillation has also been found to be fivefold higher in patients with hypokalaemia than in patients with hyperkalaemia (14). As a potentially life threatening condition, quality care of patients with hypokalaemia is paramount. With patient safety concerns identified in our hospital, quality improvement of hypokalaemia management was necessary to guide staff professional development and improve patient outcomes.

\section{Baseline measurement}

Data was collected for all medical inpatients at a district general hospital, comprising 8 acute inpatient medical wards and an emergency department (ED) managing over 30,000 cases per year. The hospital serves a catchment area of over half a million residents, many of whom are over 65 years of age. All medical inpatients over a one week period who met the criteria for hypokalaemia (serum potassium $<3.5 \mathrm{mmol} / \mathrm{L}$ on standard biochemical sample) were included in the audit. Patients were identified, prior to data collection, by obtaining a daily list of medical inpatients with confirmed serum potassium of less than or equal to $3.4 \mathrm{mmol} / \mathrm{L}$ from the biochemistry laboratory database. The patient's notes were then located and evaluated to identify if they had mild, moderate, or severe hypokalaemia. The following criteria was used:

1. Mild: $3.1-3.4 \mathrm{mmol} / \mathrm{L}$

2. Moderate: $2.5-3.0 \mathrm{mmol} / \mathrm{L}$

\section{Severe: $<2.5 \mathrm{mmol} / \mathrm{L}$}

Furthermore, those identified with hypokalaemia were analysed to identify the following:

1. Investigations requested - ECG, U\&Es, and Magnesium

2. Doses and forms of hypokalaemia management initiated (eg. tablets, elixir, or IV)

3. Prescription chart review dates for short-term hypokalaemia interventions

4. Appropriate therapeutic monitoring - Repeat serum U\&Es and ECG

A total of 32 patients (20 males, 12 females) were identified in the pre-intervention group. 25 patients met the criteria for mild hypokalaemia (3.1-3.4 mmol/L) and 7 patients met the criteria for moderate hypokalaemia $(2.5-3.0 \mathrm{mmol} / \mathrm{L})$. Only $2 / 32$ patients $(6 \%)$ had an ECG requested to monitor for cardiac arrhythmias. 13/32 $(41 \%)$ had repeat U\&Es analysed after treatment initiation. Additionally, serum magnesium levels were analysed in only $6 / 32$ (19\%) patients. Only $7 / 32(22 \%)$ patients were treated appropriately based on standard guidelines (see guideline for the management of hypokalaemia in adults). Of those patients started on hypokalaemia therapy, $8 / 17$ (47\%) had documented medication review dates. We regarded $p<0.05$ as statistically significant.

See supplementary file: ds5647.doc - "Hypokalaemia Management Guideline"

\section{Design}

It was obvious that improvement in the management of hypokalaemia was necessary. There were two important factors highlighted during the audit. One was the lack of an available trust guideline on the management of hypokalaemia coupled with the lack of understanding of appropriate medication doses for mild, moderate, and severe deficiencies. Review of guidelines for the management of hypokalaemia in adults available at other NHS trusts helped guide creation of a suitable treatment algorithm and designation of an appropriate review date (3 years) $(15,16)$.

Second was the understanding of the appropriate investigations to order when a diagnosis of hypokalaemia is confirmed and also the importance of monitoring the effect of treatment on serum potassium levels. This also includes clarifying a date for review of newly started medications. To target the above problems, the following solutions were implemented:

1. Design of an approved trust guideline on the management of hypokalaemia

2. Hypokalaemia management posters to display on all inpatient medical wards

3. Teaching sessions for junior doctors on the recognition, investigation, and management of hypokalaemia

4. Teaching sessions for nurses on the recognition, investigation, monitoring, and complications of hypokalaemia

5. Development of an easy to follow hypokalaemia 


\section{BMJ Quality Improvement Reports}

pathway/flow-chart

6. Inform medical teams about the introduction of a

hypokalaemia pathway and results of pre-intervention audit

In order to make the program sustainable, management protocol would have to be approved by the trust and incorporated into local hospital guidelines. These guidelines should be available on the hospital intranet to facilitate ease of access and healthcare professionals should be informed on their location. Alongside, junior doctors and nurses should have continuing education on the management of hypokalaemia. The aim of the audit was to introduce such guidelines and create an informative educational programme and platform for sustainable clinical improvement.

\section{Strategy}

PDSA cycle 1: A hypokalaemia management pathway was first created as this was not available in the trust. Meetings were held to design the pathway with emphasis on introducing safe clinical practice and utilising feedback to improve the guideline. Design was focused on a management algorithm that allowed easy and unambiguous interpretation by clinicians. The pathway was simple with clear differentiations between the management of mild, moderate, and severe hypokalaemia. A traffic-light colour coding system was used to highlight the separate classifications of mild (green), moderate (amber), and severe (red). A first draft of the guideline was displayed in the medical admissions unit. This received positive feedback from junior and senior doctors as it was easy to understand and clearly defined the different management options for hypokalaemia.

PDSA cycle 2: To raise awareness of hypokalaemia management, we designed treatment algorithm posters for display on all inpatient medical wards. The posters were in the format of a flow-chart to allow easy identification of the correct treatment for specific low serum potassium values. Furthermore, advice on clinical investigations and monitoring was clearly defined with escalation of care protocols evident. The design was bold and colourful with easy to read fonts and the poster received promising feedback from service users. After minor adjustments to the layout, the poster was fit for distribution to the wards.

PDSA cycle 3: Formal educational and awareness talks were given to junior doctors working in medical departments on the recognition and management of hypokalaemia. A teaching presentation was designed and multiple sessions organised for groups of 12 . The treatment algorithm was highlighted with attention given to the importance of recognising hypokalaemia, requesting appropriate investigations, and monitoring the effect of treatment. Feedback was received at the end of each session. Inclusion of hypokalaemia in the junior doctor teaching timetable and a lanyard-sized treatment card was designed in response to this.

PDSA cycle 4: The hypokalaemia treatment algorithm and a summary of the audit and pre-intervention results were emailed to all junior doctors and senior house officers working on medical wards to encourage greater awareness of areas of improvement and management options. A questionnaire filled out by recipients revealed that the lanyard-sized flow charts were a useful tool to facilitate management of hypokalaemia on call.

PDSA cycle 5: After four weeks of interventions, we conducted a reaudit over a one week period. The results were promising. Following this, the next few months were spent incorporating hypokalaemia management in the hospital guidelines and providing educational 'refresher' sessions to junior doctors, particularly as the Foundation changeover period coincided with this cycle. Meetings with consultant physicians in nephrology, acute medicine and ED were arranged to refine the treatment algorithm. After unanimous approval, the guidelines were available on the hospital intranet system.

PDSA cycle 6: Informal teaching sessions were held on each of the medical wards with nurses and pharmacists on the investigations and monitoring of hypokalaemic patients. The aim was to encourage allied healthcare professionals to assist and, if necessary, prompt doctors to consider medication review dates and regular monitoring of therapeutic interventions.

PDSA cycle 7: An audit of performance against the new hypokalaemia proforma and use of treatment algorithm commenced three months after PDSA cycles 1-6 were completed. Following the overall success of the audit, the results were presented at a trust performance meeting.

\section{Results}

Results were initially collected four weeks post-intervention, which showed dramatic improvement (see table 1.0). A total of 30 patients were identified in this post-intervention group (10 males, 20 females). The same method of daily liaison with the biochemistry department to identify hypokalaemic patients was employed. There were $16 / 30$ patients who qualified as mild hypokalaemia (3.1-3.4 $\mathrm{mmol} / \mathrm{L}$ ) and $14 / 30$ with moderate hypokalaemia (2.5-3.0 mmol/L). No patients were identified with severe hypokalaemia $(<2.5$ $\mathrm{mmol} / \mathrm{L}$ ). A frequent observation made during the pre-intervention audit was the absence of monitoring treatment with repeat U\&Es, requesting an ECG and analysing serum magnesium levels. Although the analysis of serum magnesium levels did not show a significant improvement (8/30), it was promising to see greater awareness of other investigations associated with hypokalaemia, encouraging improved patient safety.

Identification of appropriate management options for different levels of hypokalaemia and administration of correct medication doses also showed improvement but, unfortunately, despite demonstrable improved understanding of hypokalaemia management options, medication reviews were largely unchanged between the groups.

The re-audit conducted after three months, starting from May 2014 also showed sustainable improvement. In total 60 sets of patients notes coded as 'hypokalaemia' were identified and retrospectively reviewed. We decided to use coded notes to gain a higher number of cases for analysis and a retrospective approach to ensure the junior doctor base was the same, as job changeovers were due to occur at the time of re-audit. This also helped limit bias towards 
positive results secondary to junior doctors reacting to our presence on the ward. Each case identified through coding was verified against lab results during the admission to ensure they met our criteria for hypokalaemia and to verify the accuracy of coding. The results are displayed in table 1.0 with the aim of demonstrating continued recognition, investigation, and appropriate management of hypokalaemia beyond the initial audit. It is hoped that sustainability of this programme will ultimately improve patient care and reduce harm.

The requesting of an ECG prior to initiating treatment coupled with repeat U\&Es for therapeutic monitoring of potassium levels appears to show a positive trend. ECGs were also signed to indicate that they had been reviewed in a timely fashion. Furthermore, it could be argued that not all patients with mild hypokalaemia require an ECG at diagnosis unless they are a high risk candidate for cardiac arrhythmias.

Although repeat U\&Es were more frequently requested, a negative trend was observed for magnesium levels with only $25 \%$ noted to have magnesium 'added on' to biochemical tests indicating hypokalaemia. The reason for low rates of magnesium analysis is not altogether clear but it could be due to lack of education amongst junior doctors regarding the relationship between hypomagnesaemia and hypokalaemia.

The prescribing of appropriate medication doses for mild, moderate, and severe hypokalaemia shows a consistent pattern. Pleasingly, medication review dates have shown a positive trend after teaching sessions were delivered to allied healthcare professionals. We hypothesise that this improvement is likely to be due to the nurses and pharmacists challenging and encouraging junior doctors to consider highlighting treatment timelines.

Certainly pre-intervention baseline measurements on adherence to hypokalaemia guidelines were significant and suggested a strong correlation between inadequate patient management and absence of a treatment algorithm $(p<0.05)$. However, results from the postintervention group imply a more random relationship between improvement in hypokalaemia management and the introduction of teaching sessions and new clinical guidelines $(p>0.05)$. The cause of this is unknown and despite these statistical findings, quality improvement in patient care has still been observed.

See supplementary file: ds5757.doc - "Pre-intervention and postintervention results"

\section{Lessons and limitations}

We have learnt that junior doctors can instigate important change to clinical practice with small but well considered interventions. This audit has highlighted the importance of evidence based medicine and how it must continually evolve to shape and develop future practice. It was evident that with a structured teaching programme aimed at a range of healthcare professionals coupled with a clear and accessible management guideline that the investigation and treatment of hypokalaemia could be dramatically improved. It is hoped that with continued training and modification of the interventions developed during this audit that the management of hypokalaemia will continue to progress in this hospital. Furthermore, involvement of nurses and pharmacists in this process has helped to encourage doctors to challenge their own practice further and highlight the benefit of hollistic, MDT-led patient management.

Limitations of this project include small sample sizes and the lack of data regarding management of severe hypokalaemia. Potassium levels $<2.5 \mathrm{mmol} / \mathrm{L}$ are uncommon (17) and we have been unable to review how effectively these patients are managed with appropriate therapeutic interventions and escalation of care. However, given the results of this audit we anticipate good compliance with the treatment algorithm when managing severe hypokalaemia. Additionally, we have not explored the rates of complications in those patients who were managed differently to the treatment guideline. We recognise that the surgical and HDU/ICU wards were not included in this audit and this allows for future expansion of the project at a later date. Low levels of potassium in post-operative patients are common and patients with large and continuous fluid loss from the gastrointestinal tract are prone to develop hypokalaemia (18). Mechanical bowel preparations also contribute to a high incidence of hypokalaemia on the surgical wards (18). Furthermore, adverse effects of anaesthesia in patients with hypokalaemia have always been a particular clinical concern amongst anaesthetists, and pre-operative serum potassium values of less than $3.5 \mathrm{mmol} / \mathrm{L}$ are associated with a higher incidence of arrhythmias than pre-operative levels above $3.5 \mathrm{mmol} / \mathrm{L}$ (19). Therefore both surgical and anaesthetic specialities would make for interesting sub-analysis in the future.

Following this study, all junior doctors in our hospital will receive a tutorial on the management of hypokalaemia at the start of the working year. They will also receive a copy of the management guideline and information on its intranet location.

\section{Conclusion}

Through implementation of multiple interventions to improve awareness and understanding of the investigations, treatment, and monitoring of the hypokalaemic patient, we have achieved a notable improvement in management. The repeat audit has shown that the changes initiated in the post-intervention group have been maintained. This may promote better outcomes for hypokalaemic patients, reduction in complications, and a shorter treatment duration.

\section{References}

1. Paice BJ, Paterson KR, Onyanga-Omara F, Donnelly $T$, Gray JM, Lawson DH. 1986. Record linkage study of hypokalaemia in hospitalized patients. Postgrad Med J. 62(725):187-91.

2. Gennari, J.F. 1998. Hypokalemia. N Engl J Med. 339:451-458.

3. Knochel JP. 1984. Diuretic-induced hypokalemia. Am J Med. 5;77(5A):18-27. 


\section{BMJ Quality Improvement Reports}

4. Crop MJ, Hoorn EJ, Lindemas J, Zietse R. 2007. Hypokalemia and subsequent hyperkalemia in hospitalized patients. Nephrol Dial Transplant. 22:3471-3477.

5. Janko O, Seier J, Zazgornik J. 1992. Hypokalemia: incidence and severity in a general hospital [in German]. Wien Med Wochenschr. 14278- 81.

6. Widimsky, P. Hypokalaemia and the heart. E-journal of Cardiology Practice [Online] 2008;7 [accessed 23 April 2015]. Available from:

http://www.escardio.org/communities/councils/ccp/e -journal/volume7/Pages/vol7n9.aspx\#.U_d3cvldV8E

7. Paltiel O, Salakhov E, Ronen I, Berg D, Israeli A. 2001. Management of Severe Hypokalemia in Hospitalized Patients: A Study of Quality of Care Based on Computerized Databases. Arch Intern Med. 161(8):1089-1095.

8. Newmark, S.R. and Dluhy, R.G. 1975. Hyperkalemia and hypokalemia. JAMA. 231(6): pp.631-3.

9. Schulman M, Narins RG. Hypokalemia and cardiovascular disease. 1990. Am J Cardiol. 65(10):4E-9E.

10. Perazella MA, Brown E. 1994. Electrolyte and acid-base disorders associated with AIDS: an etiologic review. J Gen Intern Med. 9(4):232-6.

11. Elisaf M, Liberopoulos E, Bairaktari E, Siamopoulos K. 2001. Hypokalaemia in alcoholic patients. Drug Alcohol Rev. 21(1):73-6.

12. Al-Momen A, El-Mogy I. 2005. Intragastric balloon for obesity: a retrospective evaluation of tolerance and efficacy. Obes Surg. 15(1):101-5.

13. Kjeldsen, K. Hypokalemia and sudden cardiac death. 2010. Exp Clin Cardiol. 15(4): e96-e99.

14. Clausen TG, Brocks K, Ibsen H. 1988. Hypokalemia and ventricular arrhythmias in acute myocardial infarction. Acta Med Scand. 224(6):531-7.

15. Nottingham University Hospitals NHS Trust. Guideline for the treatment of hypokalaemia in adults. 2009. [Accessed: 5 July 2015]. Available from:

https://www.nuh.nhs.uk/handlers/downloads.ashx?id=35682

16. Gloucestershire Hospitals NHS Foundation Trust. Guideline for the management of hypokalaemia in adults. 2010. [Accessed: 5 July 2015]. Available from: http://www.gloshospitals.nhs.uk/SharePoint110/Antibiotics\% 20Web\%20Documents/TG/Hypokalaemia\%20Guidelines.pd $\underline{f}$

17. Halevy J, Gunsheroyitz M, Rosenfeld JB. 1988. Lifethreatening hypokalaemia in hospitalised patients. Miner Electrolyte Metab. 14:163-6.

18. Downing T, Bennion R, Sadeghi A. 1983. Effect of preoperative colon preparation on serum potassium. Am Surg. 49 (8):414-416.

19. Wahr J, Parks R, Boisvert D et al. 1999. Serum potassium levels and perioperative outcomes. JAMA. 281:2203-2210.
Dr Sarah Dyer - Consultant in Acute Medicine. Facilitation of guideline development and approval of teaching materials.

\section{Ethical approval}

This work is deemed an improvement study and not a study on human subjects. Local policy did not require that ethical approval be acquired before commencement of this audit.

\section{Declaration of interests}

Nothing to declare

\section{Acknowledgements}

